Results A total of 40 RCTs were included. The majority compared methadone to morphine or fentanyl. Methadone was effective in certain orthopedic, spinal, and cardiac surgeries. It was superior to fentanyl in the management of head-and-neck cancer pain. There was variability in the limited data on the management of neuropathic pain. Side effects experienced with methadone use were similar to a comparison drug.

The effectiveness of methadone in the management of postsurgical and cancer pain was dependent on the procedure and cancer type, respectively. Methadone may be useful as an adjunctive analgesic, to lower the dose of another drug

Conclusions Methadone may be a valuable in the management of post-surgical, cancer, or nociceptive pain, and in patients with renal impairment. Prescribers should consult a specialist prior to starting or discontinuing methadone.

Future Research: Reported outcomes for measuring analgesia must be standardized. Patients should be stratified by procedure and cancer type in future RCTs.

\section{ULTRASOUND GUIDED PULSED RADIOFREQUENCY (PRF) FOR THE TREATMENT OF POSTHERPETIC NEURALGIA: A CASE REPORT}

${ }^{1} \mathrm{P}$ Fusco, ${ }^{2} \mathrm{~W}$ Ciaschi, ${ }^{3} \mathrm{~F}$ Angelucci ${ }^{*},{ }^{3} \mathrm{~F}$ Marinangeli. ${ }^{1}$ Department of Anesthesia and Intensive Care, San Salvatore Academic Hospital, L'Aquila, Italy; ${ }^{2}$ Department of Anesthesia and Intensive Care, F. Spaziani Academic Hospital, Frosinone, Italy, ${ }^{3}$ University of L'Aquila, Department of Life, Health and Environmental Sciences, L'Aquila, Italy

\subsection{6/rapm-2021-ESRA.81}

Background and Aims Post-herpetic neuralgia is a persistent pain condition that occurs after an acute vesicular rash of herpes zoster heals ${ }^{1}$.The intense and disabling pain affect the patient's quality of life, his quality of sleep and his ability to perform daily activities.

This report describes a treatment case of postherpetic neuralgia with ultrasound-guided PRF.

Methods The case was a 56-year-old woman with a twomonth history of continuous pain and burning in the anterior and left thoracic region, left arm. At her first visit, the VAS was $10 / 10$. The patient was in lateral decubitus position on the operating table with slight hyperextension of the neck (figure 1). After preparation of the neck region, a sterile linear probe was placed transversely on the medial edge of the sternocleidomastoid muscle. In this position the body and the transverse process of C6 were identified. The probe was slid caudally to identify the transverse process of C7. Under

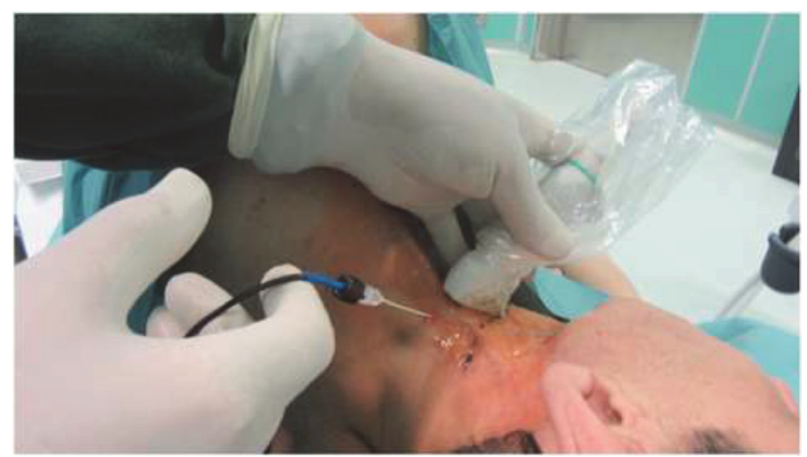

Abstract 81 Figure 1

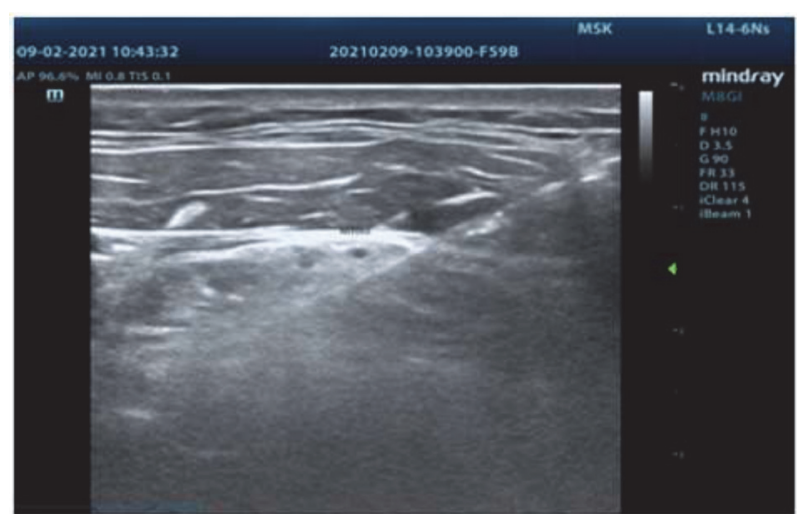

\section{Abstract 81 Figure 2}

constant ultrasound guidance and fluoroscopy, a 22 G 100 $\mathrm{mm}$ radiofrequency needle was introduced and advanced until it reached the anterior margin of the lungus colli muscle (figure 2). Subsequently, a thermocouple connected to a radiofrequency generator was inserted to reproduce the patient's paresthesia via sensitive stimulation (50 HZ), 0.7$0.9 \mathrm{mV}$. The pulsed radiofrequency treatment was performed with 1200 pulses at $42^{\circ} \mathrm{C}$.

Results At the end of the procedure the patient reported Vas 0 .

Conclusions However, further studies would be needed to assess whether the proposed technique could be considered a valid therapeutic tool for pain control.

\section{PULSED-RADIOFREQUENCY (PRF) THERAPY IN THE MANAGEMENT OF CHRONIC NEUROPHATIC PAIN DUE TO A NEUROMA FORMATION IN A NEPHROSTOMY SCAR: A CASE REPORT}

D Williams* ${ }^{*}$ B Bonet. Mid Cheshire Hospital NHS Foundation Trust, Crewe, UK

\subsection{6/rapm-2021-ESRA.82}

Background and Aims A neuroma is a mass of nerve tissue that forms at a site of injury. They can be exquisitely tender when pressure is applied, leading to a loss of function by reducing both movement and contact with the affected area. Pulsed radiofrequency (PRF) is a novel technique that is safe and effective in the management of chronic pain syndromes. However, its effectiveness in treating conditions such as painful neuromas has not been fully elicited. We present a case of a gentleman treated with PRF for a neuroma that alleviated his symptoms of significant neuropathic scar pain.

Methods We assessed this gentleman's pain by assessing his Visual Analogue Scale (VAS), reviewed previous management strategies, current medication and impact of the condition on his life. A single session of ultrasound guided PRF was then delivered to the neuroma.

Results A 66-year old gentleman presented with an 8-year history of persistent neuropathic pain in his right loin associated with a nephrectomy scar despite analgesic medication (oral and transdermal) and trigger point injections. Pain was $7 / 10$ on the VAS scale with significant impact on the patient's psychological wellbeing. After PRF, his pain score improved 
ceased analgesic medication and remained pain free at 18month follow-up.

Conclusions Neuropathic pain is a difficult condition to manage due to the biopsychosocial factors involved whereby diverse strategies may have to be utilized. PRF has been shown to be successful in the treatment of a painful neuroma following scar formation and so offers an alternative, medication-free approach to treating this condition.

\section{Miscellaneous}

\section{ULTRASOUND-GUIDED NERVE BLOCK WITH TELEMEDICINE}

S Oku*, Y Mizuno, T Goto. Yokohama City University, Yokohama, Japan

\subsection{6/rapm-2021-ESRA.83}

Background and Aims New skills and knowledges for various ultrasound guided nerve blocks (UGNB) have been developed. Instructions from experienced expert may be essential for the education, however, these chances are limited partly because of availability of the expert instructor.

In this study, we developed telemedicine system for UGNB instruction and investigated whether the system could be useful practically for remote UGNB in 5 patients.

Methods At a practitioner site, video conference device (SONY) with ultrasound machine (Edge, Sonosite) and field camera was set in Sado General Hospital. At an instructor site, the device with annotation tablet and monitors of the ultrasound and the field was set in Yokohama City University Hospital $300 \mathrm{~km}$ away (figure 1). Ethics Committee approval and informed consent by the patients were obtained.

Results Two lateral transversus abdominis plane blocks, two rectus sheath blocks and a pectoral nerves II block were performed in 5 patients underwent open inguinal hernia repair, open umbilical hernia repair and mastectomy, respectively. At instructor site, instructor added annotation on ultrasound video, and field camera monitor displayed practitioner's

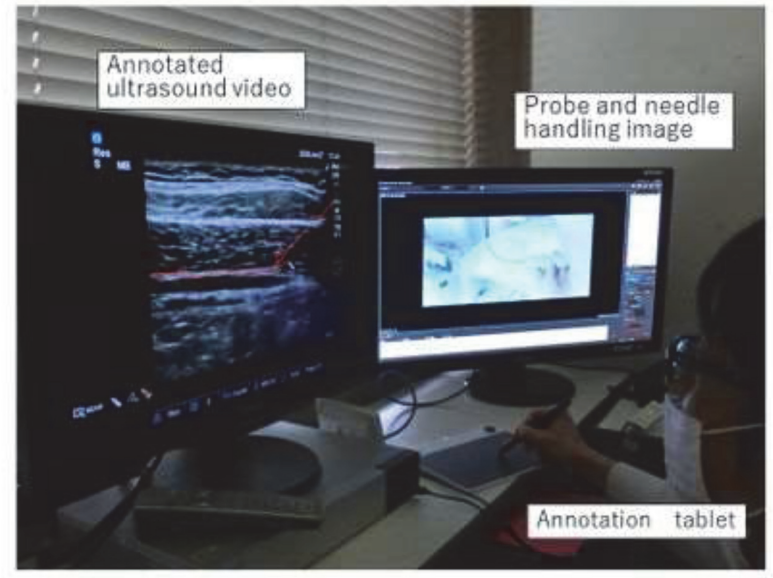

Abstract 83 Figure 2

handling of the probe and patient position. (figure 2)These simultaneous monitorings of ultrasound and field allowed instructor to provide prompt supports and ensure procedural safety by double check. These UGNB procedures were performed in 5-10 minutes without any clinical and mechanical problem.

Conclusions We developed telemedicine system for remote instruction of UGNB. This system could be used practically in clinical cases and might be useful to improve safety.

\section{THORACIC INJURY PATHWAY TO OPTIMISE PAIN \& PHYSIOTHERAPY}

S Mohamedally*, B Fox. Queen Elizabeth Hospital Kings Lynn, King's Lynn, UK

\subsection{6/rapm-2021-ESRA.84}

Background and Aims

- Evaluate the referral of rib fracture patients to anaesthetics/ acute pain team/physiotherapy \& Critical Care Outreach Team (CCOT)

\section{Telemedicine system for UGNB}

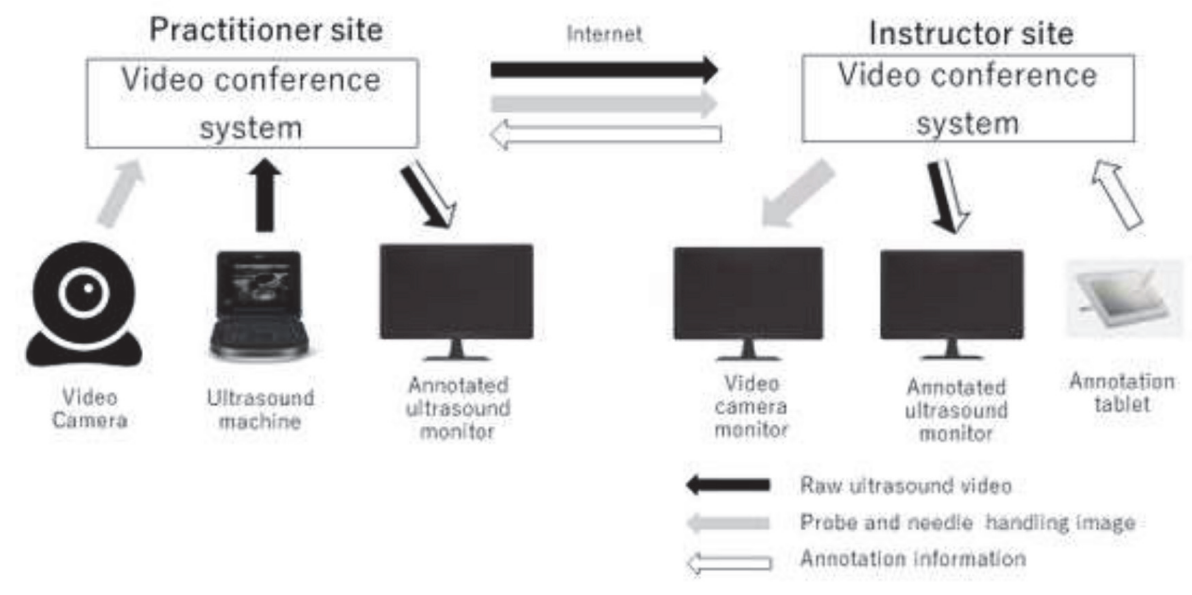

Abstract 83 Figure 1 